\title{
Application of Transfer Matrix Approach to Modeling and Decentralized Control of Lattice-based Structures
}

\author{
Nick Cramer*, Sean Shan-Min Swei; Kenny Cheung ${ }^{\ddagger}$ and Mircea Teodorescu ${ }^{\S}$
}

\begin{abstract}
This paper presents a modeling and control of aerostructure developed by lattice-based cellular materials/components. The proposed aerostructure concept leverages a building block strategy for lattice-based components which provide great adaptability to varying flight scenarios, the needs of which are essential for in-flight wing shaping control. A decentralized structural control design is proposed that utilizes discrete-time lumped mass transfer matrix method (DT-LM-TMM). The objective is to develop an effective reduced order model through DT-LM-TMM that can be used to design a decentralized controller for the structural control of a wing. The proposed approach developed in this paper shows that, as far as the performance of overall structural system is concerned, the reduced order model can be as effective as the full order model in designing an optimal stabilizing controller.
\end{abstract}

\section{Introduction}

Earlier research studies conducted by NASA had shown that active control of wing twist and deflection at local wing sections can improve overall aerodynamic efficiency $102[3]$ Two recent NASA projects to realize in-fight aeroelastic wing shape control are the Variable Camber-Continuous Trailing Edge Flap (VCCTEF) and Distributed Electric Propulsion (DEP) concepts. The VCCTEF strategy divides a trailing edge into many flap sections, each of which can be individually controlled subject to the constraints of an interstitial elastomeric interpolating surface. Chord-wise and span-wise adaptive wing shaping for various flight scenarios can be achieved in this manner! DEP proposes to accomplish similar shape morphing modes, but with differential control of spatially distributed electric propulsion systems.

The cellular composite materials proposed in this paper allow for high-performance structures to be incrementally assembled, modified, and repaired without requiring custom tooling. Fiber composites have been widely used to construct truss cores ${ }^{\sqrt[5]{5}}$ and structural frames ${ }^{6}$ These have all entailed the development of processes to continuously wind the fibers through a structure, and subsequently cure a resin matrix around them. Instead, the approach taken here uses parts with carbon-fiber beams and loops that are reversibly linked. These materials are assembled from mass-produced carbon fiber reinforced polymer parts with integrated interlocking connections.

Modeling of high dimensional lattice structures using conventional FEM approach can be a challenge, mainly because it is difficult to analyze and visualize the integrated lattice structure in real-time. In this paper, we propose to use the concept of discrete-time lumped mass transfer matrix method (DT-LM-TMM) to model and analyze large dynamical systems. Due to the unique construction of the wing, as shown in Figure 1, we believe that the lumped mass approach will be appropriate with masses being located at the ribs and the spring being calibrated to the stiffness of the wingbox in between. The damping that is included is a combination of the extremely low internal damping of the carbon fiber and the friction due to the reversible attachment of the parts. The basic concept behind this approach was inspired by the work of Tan et al., ${ }^{7}$ where the notion of modified transfer matrix method (M-TMM) approach was developed by utilizing the dynamic stiffness matrix of finite element. The primary goal there was to reduce the computational efforts involved in structural analysis. In this paper, in addition to incorporate the notion of M-TMM, we also utilize the numerical integration approach proposed in Kumar and Sankar ${ }^{8}$ and develop a reduced order

\footnotetext{
${ }^{*}$ Graduate Student, Computer Engineering Department, University of California at Santa Cruz

$\dagger$ Research Scientist, Intelligent Systems Division, NASA Ames Research Center

$¥$ Aerospace Engineer, NASA Ames Research Center

$\S$ Adjunct Associate Professor, Computer Engineering Department, University of California at Santa Cruz
} 


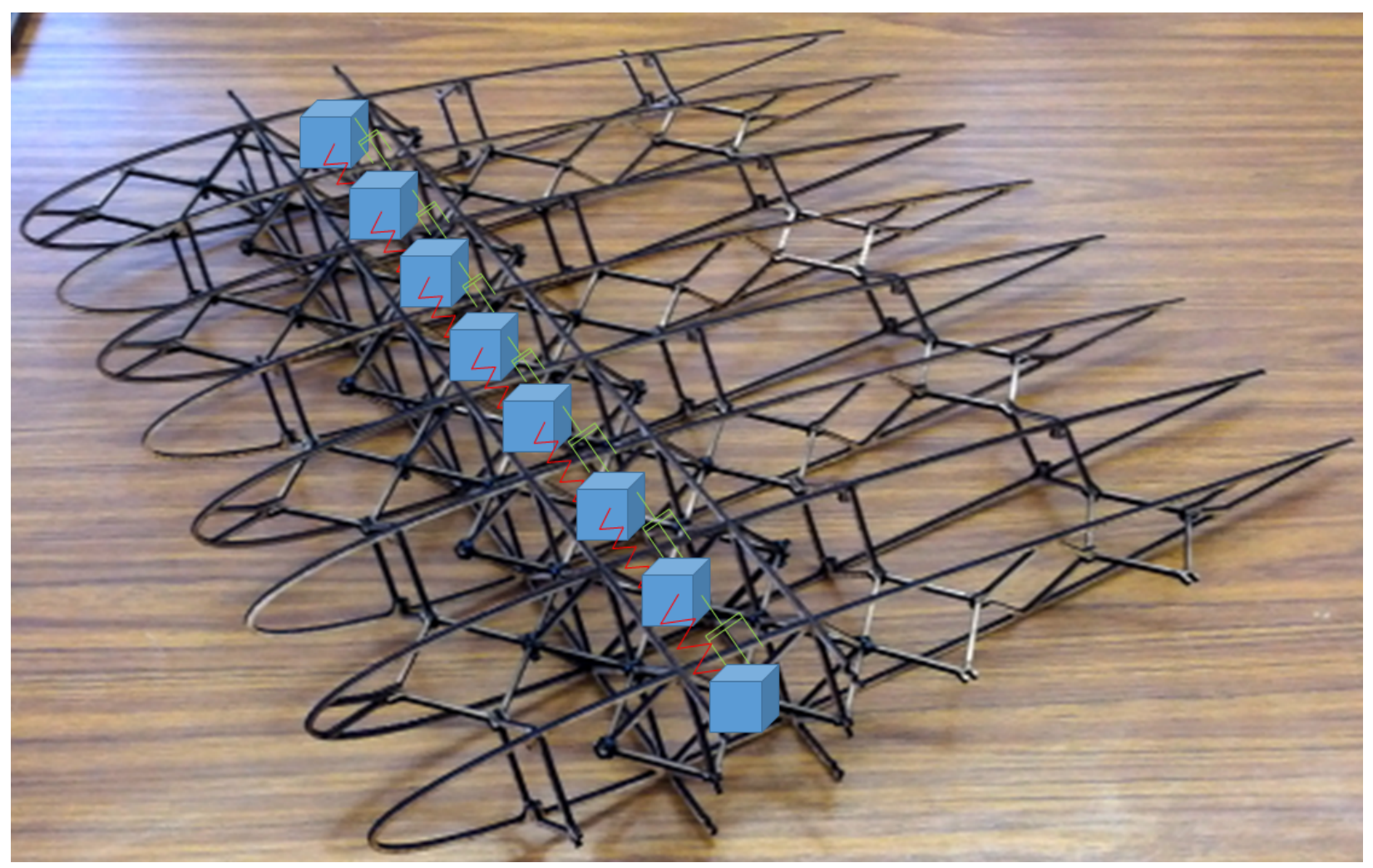

Figure 1. Lattice-based composite cellular wing structure and its lumped mass model.

discrete-time state-space model that is best suited for control synthesis. An optimal decentralized LQR controller is then designed for the reduced order model, and it is demonstrated that the overall structural system performance is comparable with that of the optimal LQR controller with full order model.

This paper is organized as follows. In Section II we provide a brief overview of lattice-based cellular materials/components. Section III contains the introduction and some basics of discrete-time transfer matrix method, and the reduced-order model by following the proposed transfer matrix method is given in Section IV. Section V focuses on developing and formulating the decentralized controls, and Section VI contains numerical simulations and discussions. Concluding remarks are made in Section VII.

\section{Lattice-based Cellular Structures}

Cellular materials can incorporate multiple functions; these have conventionally been based on uniform periodic or stochastic structures ${ }^{[9}$ The discrete construction of digital composites introduces an independent design degree of freedom that allows global functional properties to be determined by the local placement of heterogeneous components. The lattice-based cellular structures will be assembled in a regular fashion from many discrete components, for which there is a small set of different types. These can be made and measured separately, and then combined in a hierarchical fashion, based on their discretization. Preliminary tests indicate that the macroscopic properties of an assembled structure can be accurately predicted from the behavior of its components, by using the topological connectivity of the lattice to define an equivalent mesh for modeling, and by using measurement of individual components to define the behavior of the mesh elements. The discrete construction of these materials will allow for combinatorial optimization of system designs. 


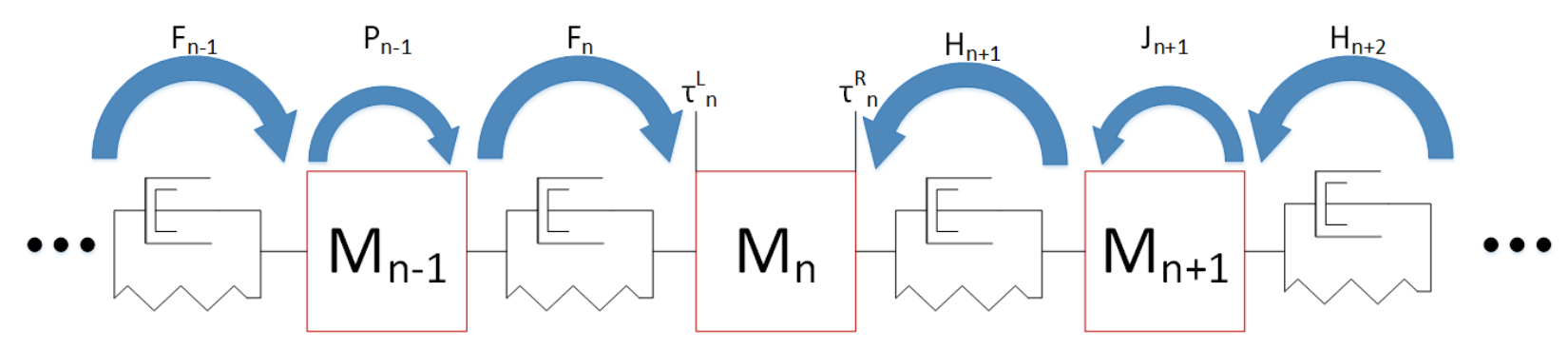

Figure 2. Example of a lumped mass configuration for discrete-time transfer matrix method.

\section{Discrete Time Transfer Matrix Method}

For completeness we will provide a quick overview of the discrete time transfer matrix method provided by Kumar and Sankar ${ }^{8}$ before going into the adaptations for use in decentralized control. For a lumped mass model; see Figure 2, the equation of motion for a given subsystem $n$ can be described by

$$
m_{n}\left(t_{i}\right) \ddot{x}_{n}\left(t_{i}\right)=\tau_{n}^{R}\left(t_{i}\right)-\tau_{n}^{L}\left(t_{i}\right)+f_{n}\left(t_{i}\right)
$$

where $\tau_{n}^{R}$ and $\tau_{n}^{L}$ denote the forces from right and left side of $n$, and $f_{n}$ the control force at $n$. Furthermore, $\tau_{n}^{L}$ can be described by

$$
\tau_{n}^{L}=k_{n}\left(t_{i}\right)\left[x_{n}^{L}\left(t_{i}\right)-x_{n}^{R}\left(t_{i}\right)\right]+c_{n}\left(t_{i}\right)\left[\dot{x}_{n}^{L}\left(t_{i}\right)-\dot{x}_{n-1}^{R}\left(t_{i}\right),\right]
$$

and $\left(k_{n}, c_{n}\right)$ denote the pair of stiffness and damping components at the left side of $n$. Because of repetitiveness of the construction of subsystems, the left side forces $\tau_{n}^{L}$ at $n^{\text {th }}$ subsystem are equal to the right side forces $\tau_{n-1}^{R}$ at $(n-1)^{t h}$. Adjacently we know that there is no change in displacement across the mass. These conditions are represented as follows,

$$
\begin{gathered}
\tau_{n}^{L}=\tau_{n-1}^{R} \\
x_{n}^{R}=x_{n}^{L}
\end{gathered}
$$

The generalized form of acceleration and velocity can be represented as

$$
\begin{aligned}
& \ddot{x}_{n}\left(t_{i}\right)=A_{n}\left(t_{i}\right) x_{n}\left(t_{i}\right)+B_{n}\left(t_{i}\right) \\
& \dot{x}_{n}\left(t_{i}\right)=D_{n}\left(t_{i}\right) x_{n}\left(t_{i}\right)+E_{n}\left(t_{i}\right)
\end{aligned}
$$

where the format of $\left(A_{n}, B_{n}, D_{n}, E_{n}\right)$ depends on the chosen numerical integration scheme. Now, substituting (3) and (4) into (1) yields

$$
m_{n}\left[A_{n} x_{n}+B_{n}\right]=\tau_{n}^{R}-\tau_{n}^{L}+f_{n}
$$

where

$$
\tau_{n}^{L}=k_{n}\left[x_{n}^{L}-x_{n-1}^{R}\right]+c_{n}\left[\left(D_{n} x_{n}+E_{n}\right)^{L}-\left(D_{n-1} x_{n-1}+E_{n-1}^{R}\right)\right] .
$$

Furthermore, by combining (5) and (3b), we can rewrite (5) in the matrix representation as

$$
\left\{\begin{array}{l}
x \\
\tau \\
1
\end{array}\right\}_{n}^{R}=\left[\begin{array}{ccc}
1 & 0 & 0 \\
m_{n} A_{n} & 1 & m_{n}-f_{n} \\
0 & 0 & 1
\end{array}\right]\left\{\begin{array}{l}
x \\
\tau \\
1
\end{array}\right\}_{n}^{L}
$$

which can be described in compact form as

$$
v_{n}^{R}=P_{n} v_{n}^{L} .
$$


The same process can be applied to (3a) and (6) which results in

$$
\left\{\begin{array}{l}
x \\
\tau \\
1
\end{array}\right\}_{n}^{L}=\left[\begin{array}{ccc}
\frac{k_{n}+c_{n} D_{n-1}^{R}}{k_{n}+c_{n} D_{n}^{L}} & \frac{1}{k_{n}+c_{n} D_{n}^{L}} & \frac{-c_{n}\left(E_{n}^{L}-E_{n-1}^{R}\right)}{k_{n}+c_{n} D_{n}^{L}} \\
0 & 1 & 0 \\
0 & 0 & 1
\end{array}\right]\left\{\begin{array}{l}
x \\
\tau \\
1
\end{array}\right\}_{n-1}^{R},
$$

and therefore can be equivalently written as

$$
v_{n}^{L}=F_{n} v_{n-1}^{R} .
$$

Equations $(8)$ and $(10)$ can be combined to propagate the "state vector" $v$ spatially forward from the left boundary condition, and can be represented by

$$
\left\{\begin{array}{l}
x \\
\tau \\
1
\end{array}\right\}_{n}^{L}=\left[\begin{array}{ccc}
1 & 0 & 0 \\
-m_{n} A_{n} & 1 & -m_{n}+f_{n} \\
0 & 0 & 1
\end{array}\right]\left\{\begin{array}{l}
x \\
\tau \\
1
\end{array}\right\}_{n}^{R}
$$

or equivalently as

$$
v_{n}^{L}=J_{n} v_{n}^{R} .
$$

Similarly, Equation (3a) together with (6) results in

$$
\left\{\begin{array}{l}
x \\
\tau \\
1
\end{array}\right\}_{n}^{R}=\left[\begin{array}{ccc}
\frac{k_{n}+c_{n} D_{n-1}^{R}}{k_{n}+c_{n} D_{n}^{L}} & \frac{1}{k_{n}+c_{n} D_{n}^{L}} & \frac{-c_{n}\left(E_{n}^{L}-E_{n+1}^{R}\right)}{k_{n}+c_{n} D_{n}^{L}} \\
0 & 1 & 0 \\
0 & 0 & 1
\end{array}\right]\left\{\begin{array}{l}
x \\
\tau \\
1
\end{array}\right\}_{n+1}^{L},
$$

or can be equivalently described as

$$
v_{n}^{R}=H_{n} v_{n+1}^{L} .
$$

Figure 2 shows a sequence of lumped masses which are interconnected by stiffness and damping components modeled by spring and dashpot. The propagation of neighboring forces from both sides of the subsystem $n$ is highlighted.

\section{Modeling and Control of Lattice Structures}

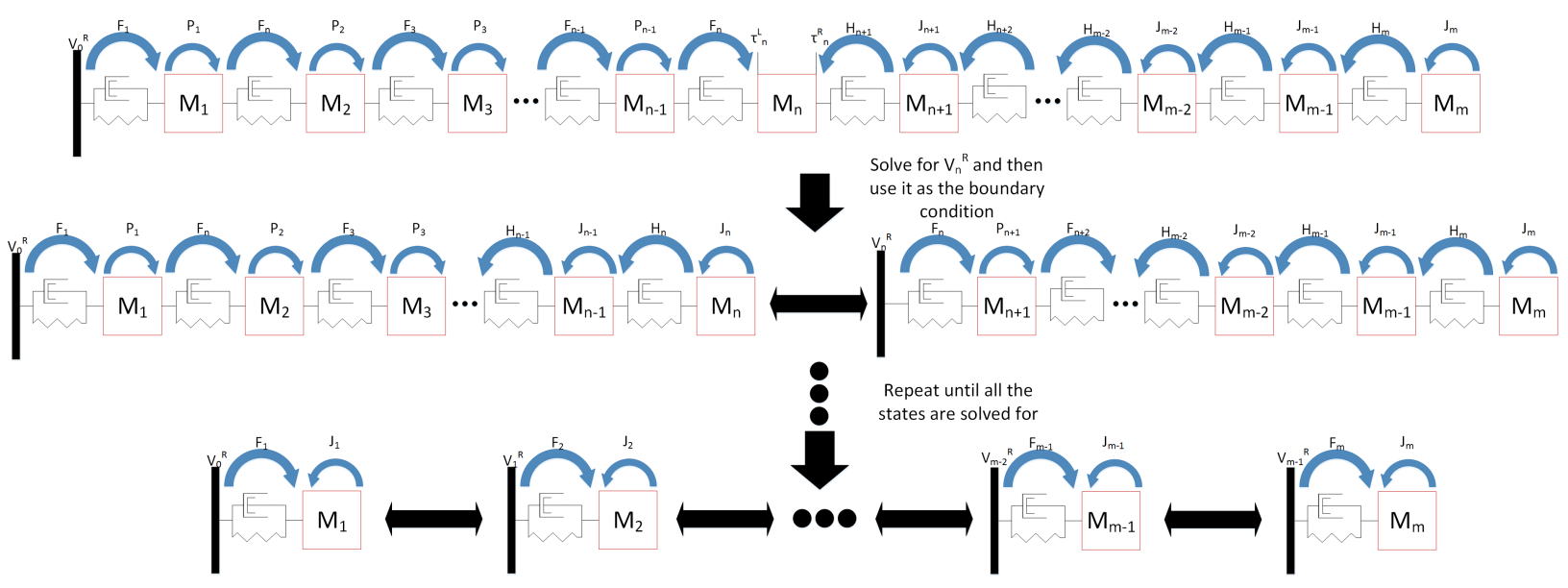

Figure 3. The combination of left and right propagation.

In this section, we make sure of the discrete-time lumped mass transfer matrix method to develop a control-centric reduced order model for control system synthesis. Figure 1 shows a section of aircraft wing built by utilizing the lattice-based cellular structure concept. The composition of lattice-based construction renders itself naturally to lumped mass system setup. Each section of airfoil provides a logical location of a lumped mass, and the connecting components between each airfoil can be modeled as spring and damper. 


\section{A. Relating node 0 to node $n$}

In this section, we work to develop a relationship between the nodal states of $n$ and 0 (the first element on the left edge). We start from (3b) and apply it to (8) and (10) to render

$$
v_{n}^{R}=P_{n} F_{n} v_{n-1}^{R},
$$

and we can continue this process until we reach subsystem $n$,

$$
v_{n}^{L}=F_{n} Q_{n} v_{0}^{R},
$$

where

$$
Q_{n}=\prod_{i=0}^{n} P_{i} F_{i}
$$

and $Q_{n}$ denotes the transfer function relating node 0 to node $n$. Note that $v_{0}^{R}$ represents boundary conditions at the left edge.

\section{B. Relating node $m$ to node $n$}

Last section we have propagated the nodal states from left to right and arrived at node $n$. In this section, we establish the relationship between node $m$ and node $n$ by propagating from right to left. Again, we start from (3b) but this time we apply it to Equations (12) and (14) to attain

$$
v_{n}^{R}=H_{n+1} J_{n+1} v_{n+1}^{R},
$$

and this process can be continued and eventually results in

$$
v_{n}^{R}=T_{n} v_{m}^{R},
$$

where $v_{m}^{R}$ is the boundary conditions at right edge, and

$$
T_{n}=\prod_{i=m}^{n} H_{i} J_{i},
$$

and $T_{n}$ represents the transfer function from node $m$ to node $n$.

\section{Combining left and right propagation}

The proposed DT-LM-TMM approach described earlier can be applied recursively, and by combining the process shown in Sections $\mathrm{A}$ and $\mathrm{B}$ yields

$$
\begin{gathered}
v_{n}^{L}=F_{n} Q_{n} v_{0}^{R} \\
v_{n}^{R}=T_{n} v_{m}^{R} \\
m\left(A_{n} x_{n}+B_{n}\right)=\tau_{n}^{R}-\tau_{n}^{L}+f_{n}
\end{gathered}
$$

We can solve 21) for the state vector $v_{n}^{R}$ at subsystem $n$. Then, by treating $v_{n}^{R}$ as the right and left boundary conditions in Equations (16) and (19), respectively, we can solve for each subsequent nodal states recursively, as illustrated in Figure 3 for the case of clamped-free boundary conditions. Given the state vector $v_{n}^{R}$, the condition (3b can be applied that allows the free-end condition on the left side, while the clamp is being applied to the right side. It should be noted that it is not necessary to solve for the internal forces if they are not of interest. 


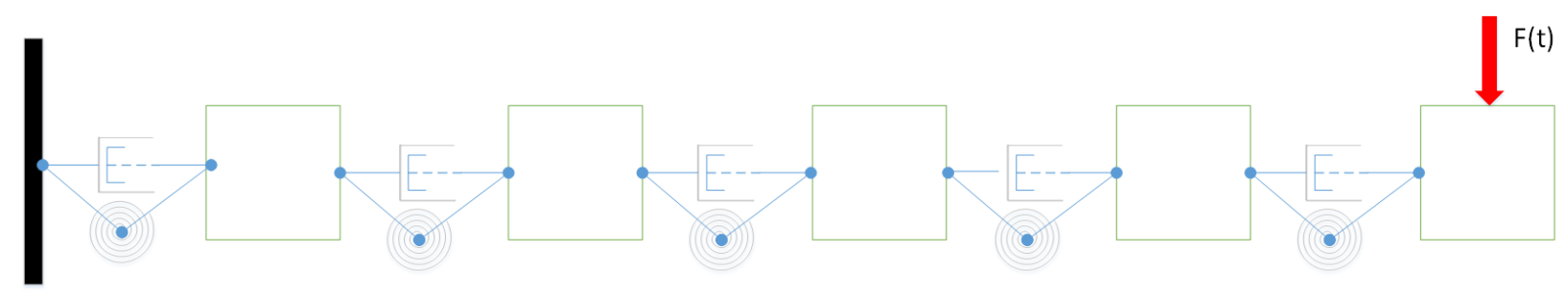

Figure 4. Five lumped mass system subject to a unit impulse.

\section{Decentralized Control Problem Formulation}

The DT-LM-TMM presented in Section C can be applied to generate full state dynamic structural responses, as was studied in Kumar and Sankar! The simulation results will be presented in the next section. The focus of this section is to develop a control-centric reduced order dynamic model that is best suited for discrete-time decentralized control design. To this end, we only consider the lumped mass systems with clamped-free boundary conditions, which models an aircraft wing attached to a fuselage. We start with a small variation of the system of equations 21 :

$$
\begin{gathered}
{\left[\begin{array}{ccc}
Q_{11} & Q_{12} & Q_{13} \\
Q_{21} & Q_{22} & Q_{23} \\
0 & 0 & 1
\end{array}\right]\left[\begin{array}{c}
0 \\
\tau_{0} \\
1
\end{array}\right]=} \\
{\left[\begin{array}{ccc}
T_{11} & T_{12} & T_{13} \\
T_{21} & T_{22} & T_{23} \\
0 & 0 & 1
\end{array}\right]\left[\begin{array}{ccc}
G_{11} & G_{12} & G_{13} \\
G_{21} & G_{22} & G_{23} \\
0 & 0 & 1
\end{array}\right]\left[\begin{array}{ccc}
H_{11}^{n} & H_{12}^{n} & H_{c}^{n}\left(E_{n-1}-E_{n}\right) \\
0 & 1 & 0 \\
0 & 0 & 1 \\
0 \\
1
\end{array}\right]=\left[\begin{array}{ccc}
F_{11}^{n+1} & F_{12}^{n+1} & F_{c}^{n}\left(E_{n}-E_{n+1}\right) \\
0 & 1 & 0 \\
0 & 0 & 1
\end{array}\right]\left[\begin{array}{c}
x_{n}^{L} \\
\tau_{n}^{L} \\
1
\end{array}\right]} \\
m\left(A_{n} x_{2}+B_{n}\right)=\tau_{n}^{R}-\tau_{n}^{L}+f_{n}
\end{gathered}
$$

By splitting the propagation to each side of the equations we are able to avoid some numerical issues and the problem becomes easier to solve. What is more important though is the explicit statement of $B_{n}$ and $E_{n}$ which we use to generate the previous states and formulate the discrete time decentralized control problem. In solving (22), we are able to attain an equation of motion that is based solely on the current and the previous conditions, as shown below

$$
C_{x_{n}^{R}} x_{n}^{R}=C_{E_{n-1}} E_{n-1}+C_{E_{n}} E_{n}+C_{E_{n+1}} E_{n+1}+C_{R}+C_{L}-m_{2} B_{n}+f_{n}
$$

where

$$
\begin{gathered}
C_{x_{n}^{R}}=\frac{G_{21} H_{11}^{n} Q_{12}-G_{11} H_{11}^{n} Q_{22}}{G_{12} Q_{22}-G_{22} Q_{12}+G_{11} H_{12}^{n} Q_{22}-G_{21} H_{12}^{n} Q_{12}}+m_{2} A_{2}+\frac{F_{11}^{n+1} R_{11} T_{21}-F_{11}^{n+1} R_{21} T_{11}}{R_{12} T_{21}-R_{22} T_{11}+F_{12}^{n+1} R_{11} T_{21}-F_{12}^{n+1} R_{21} T_{11}} \\
C_{E_{n-1}}=\frac{-G_{11} H_{c}^{n} Q_{22}+G_{21} H_{c}^{n} Q_{12}}{G_{12} Q_{22}-G_{22} Q_{12}+G_{11} H_{12}^{n} Q_{22}-G_{21} H_{12}^{n} Q_{12}} \\
C_{E_{n}}=\frac{G_{11} H_{c}^{n} Q_{22}-G_{21} H_{c}^{n} Q_{12}}{R_{12} T_{21}-R_{22} T_{11}+F_{12}^{n+1} R_{11} T_{21}-F_{12}^{n+1} R_{21} T_{11}}-\frac{F_{12}^{n+1} R_{21} T_{12}-F_{22}^{n+1} R_{12} T_{21}}{G_{12} Q_{22}-G_{22} Q_{12}+G_{11} H_{12}^{n} Q_{22}-G_{21} Q_{12}} \\
C_{E_{n+1}}=\frac{F_{c}^{n+1} R_{11} T_{21}-F_{c}^{n+1} R_{21} T_{11}}{R_{12} T_{21}-R_{22} T_{11}+F_{12}^{n+1} R_{11} T_{21}-F_{12}^{n+1} R_{21} T_{11}} \\
C_{R}=\frac{R_{23} T_{11}-R_{13} T_{21}-T_{11} T_{23}+T_{13} T_{21}}{R_{12} T_{21}-R_{22} T_{11}+F_{12}^{n+1} R_{11} T_{21}-F_{12}^{n+1} R_{21} T_{11}} \\
C_{L}=\frac{Q_{12} Q_{23}-Q_{13} Q_{22}+G_{13} Q_{22}-G_{23} Q_{12}}{G_{12} Q_{22}-G_{22} Q_{12}+G_{11} H_{12}^{n} Q_{22}-G_{21} H_{12}^{n} Q_{12}}
\end{gathered}
$$

Kumar and Sankar ${ }^{8}$ provided a comprehensive list of numerical integration techniques, including Fox-Euler, Newmark $\beta$, and Houbolt methods. In this paper, we make use of the Houbolt integration method because 
its framework can be readily incorporated into formulating the discrete-time control problem. The Houbolt integration coefficients are given by ${ }^{\sqrt{8}}$

$$
\begin{gathered}
A_{n}\left(t_{i}\right)=\frac{2}{\Delta T^{2}} \\
B_{n}\left(t_{i}\right)=-\frac{1}{\Delta T^{2}}\left[5 x\left(t_{i-1}\right)-4 x\left(t_{i-2}\right)+x\left(t_{i-3}\right)\right] \\
D_{n}\left(t_{i}\right)=\frac{11}{6 \Delta T} \\
E_{n}\left(t_{i}\right)=-\frac{1}{6 \Delta T}\left[18 x\left(t_{i-1}\right)-9 x\left(t_{i-2}\right)+2 x\left(t_{i-3}\right)\right]
\end{gathered}
$$

It is important to note that the Houbolt integration method is only valid after 3 time steps have passed. Kumar and Sankar provided an extended set of coefficients for the earlier time steps, but they are not particularly important from the controls point of view and thus ignored here.

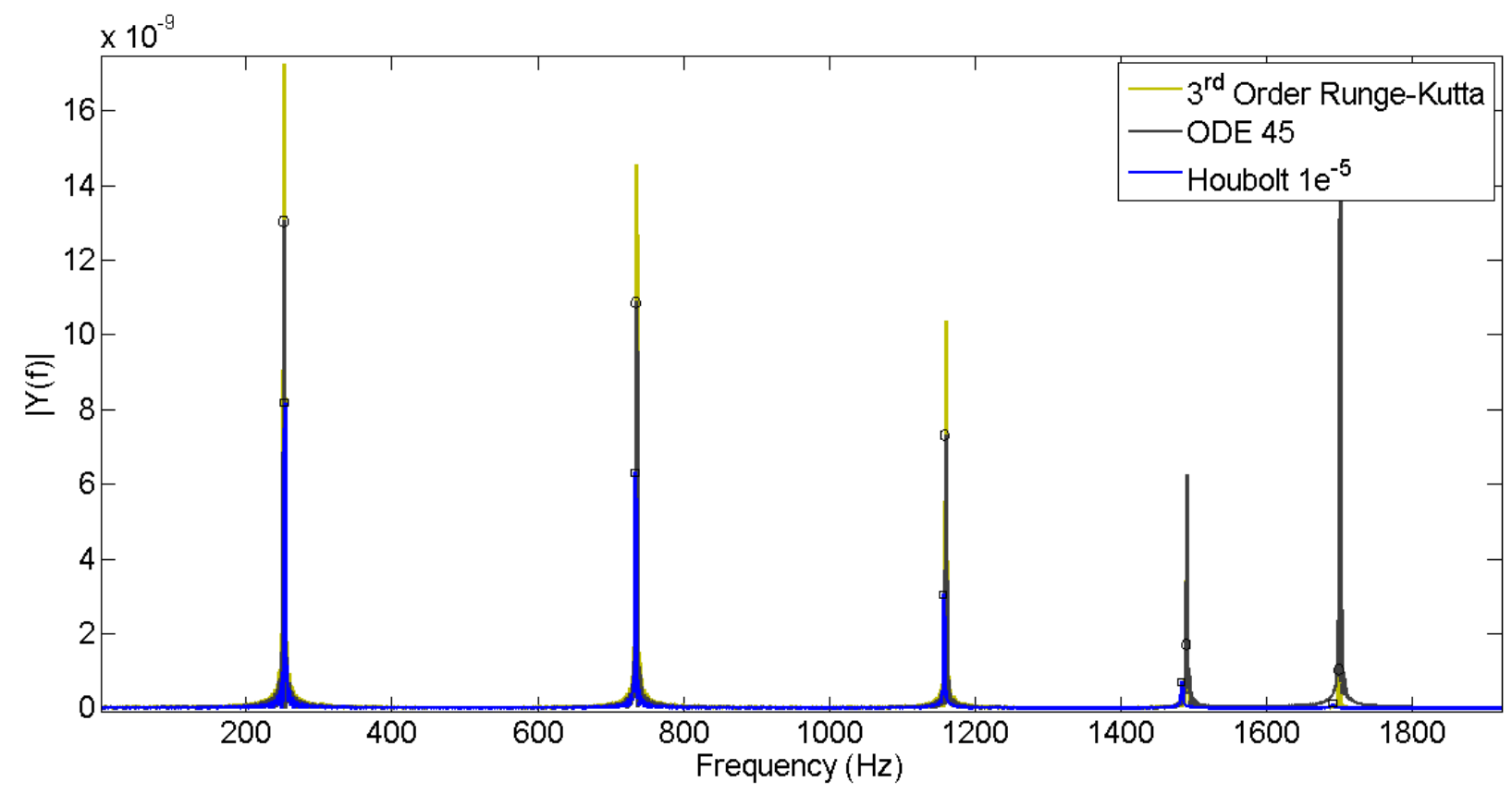

Figure 5. Fast Fourier Transforms of the third mass. The circles and squares indicate the peak magnitude.

Substituting (25) into (23) results in a discrete-time model which has smaller dimension, as shown below

$$
\begin{gathered}
x_{n}^{R}\left(t_{i}\right)=\left[\begin{array}{ccc}
\frac{1}{C_{x_{n}}}\left(\frac{-3 C_{E_{n}}}{\Delta T}+\frac{5 m_{n}}{\Delta T^{2}}\right) & \frac{1}{C_{x_{n}^{R}}}\left(\frac{3 C_{E_{n}}}{2 \Delta T}+\frac{-4 m_{n}}{\Delta T^{2}}\right) & \frac{1}{C_{x_{n}^{R}}}\left(\frac{-C_{E_{n}}}{3 \Delta T}+\frac{m_{n}}{\Delta T^{2}}\right) \\
1 & 0 & 0
\end{array}\right]\left[\begin{array}{l}
x_{n}^{R}\left(t_{i-1}\right) \\
x_{n}^{R}\left(t_{i-2}\right) \\
x_{n}^{R}\left(t_{i-3}\right)
\end{array}\right] \\
+\frac{1}{C_{x_{n}^{R}}}\left(C_{E_{n-1}} E_{n-1}+C_{E_{n+1}} E_{n+1}+C_{R}+C_{L}+f_{n}\right)
\end{gathered}
$$

which can be equivalently rewritten as

$$
X\left(t_{i}\right)=A X\left(t_{i-1}\right)+B\left(\alpha+f_{n}\right),
$$

where the system matrices $(A, B)$ and the exogenous input $\alpha$ are given by

$$
\begin{gathered}
A=\left[\begin{array}{ccc}
\frac{1}{C_{x_{n}^{R}}}\left(\frac{-3 C_{E_{n}}}{\Delta T}+\frac{5 m_{n}}{\Delta T^{2}}\right) & \frac{1}{C_{x_{n}^{R}}}\left(\frac{3 C_{E_{n}}}{2 \Delta T}+\frac{-4 m_{n}}{\Delta T^{2}}\right) & \frac{1}{C_{x_{n}^{R}}}\left(\frac{C_{E_{n}}}{3 \Delta T}+\frac{m_{n}}{\Delta T^{2}}\right) \\
1 & 0 & 0 \\
0 & 1 & 0
\end{array}\right], B=\left[\begin{array}{c}
\frac{1}{C_{x_{n}^{R}}} \\
0 \\
0
\end{array}\right] \\
\alpha=C_{E_{n-1}} E_{n-1}+C_{E_{n}} E_{n}+C_{R}+C_{L}
\end{gathered}
$$


Equation 27) is the equation of motion for the mass $m_{n}$, where $\alpha$ consists of the contribution of forces from neighboring masses and $f_{n}$ is the control input applied to mass $m_{n}$. To design a stabilizing decentralized controller, we consider $\alpha$ to be a "weak" coupling between mass $m_{n}$ and its neighbors, and therefore we can treat mass $m_{n}$ as a local subsystem! 10

It is important to note that $Q_{i, i}, T_{i, i}$, and $G_{i, i}$ contain information on the configuration of the spring, mass, and damper systems that were present between subsystem $n$ and the boundary conditions. The configuration of $C_{x_{n}^{R}}$ and $C_{E_{n}}$ also change depending on the boundary conditions. This allows the decentralized subsystem $m_{n}$ to act as if it is directly connected to the boundary conditions through a series of combined mass, spring, damper systems.

\section{Results and Discussion}

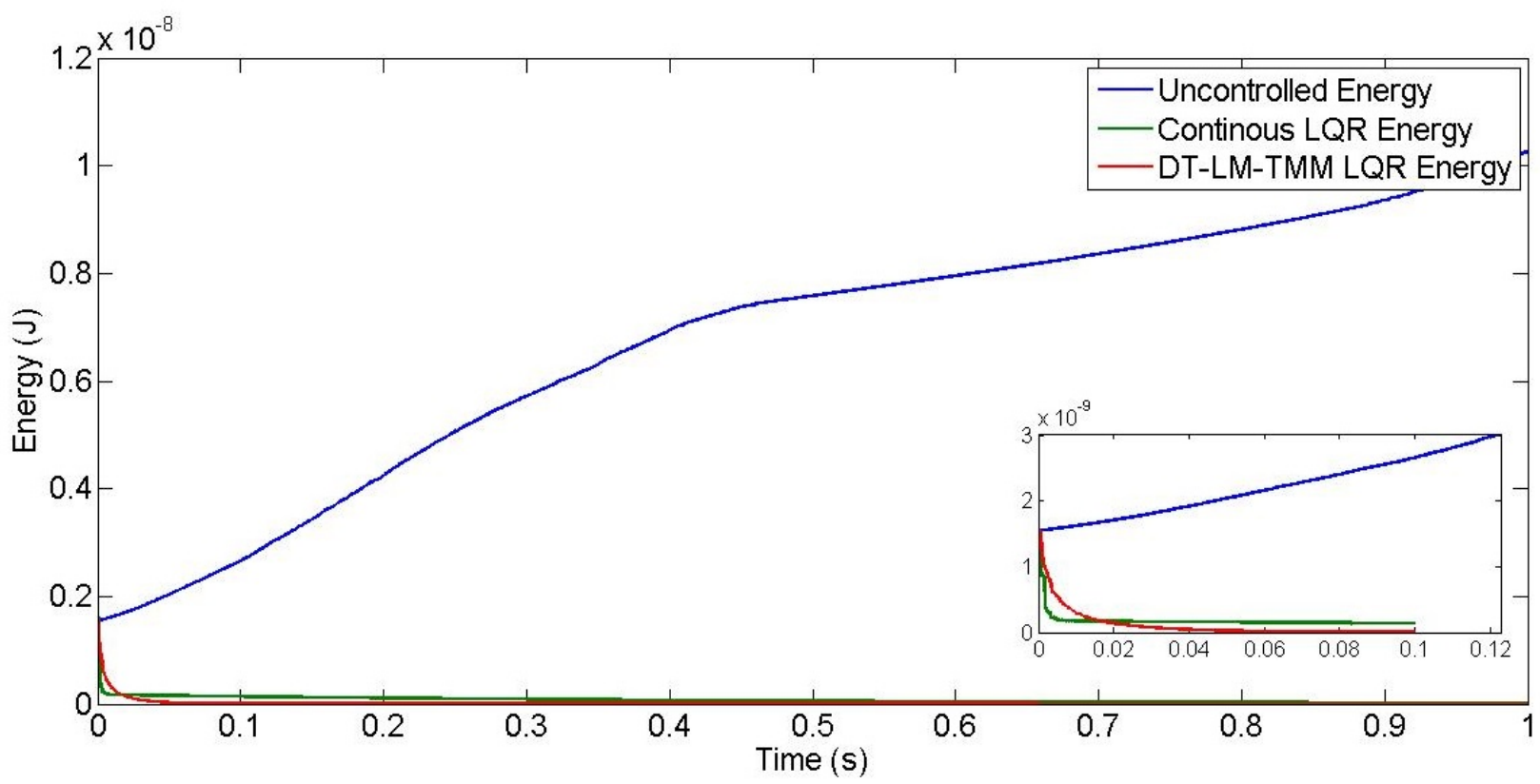

Figure 6. Comparison of total energy of the uncontrolled, continuous LQR controlled, and DT-LM-TMM decentralized controlled systems.

To demonstrate the proposed DT-LM-TMM and the decentralized control concept developed in the previous section, here we consider the vibration control of a five lumped mass system with clamped-free boundary conditions subject to a unit impulse, as shown in Figure 4. The control force is applied at the third mass.

Throughout this section, the parameters used for generating simulation results are given in Table 1 We first perform a frequency domain analysis for the lumped mass system using three different numerical integration schemes; namely, 3rd order Runge-Kutta, 4th/5th order ODE, and Houbolt. Figure 5 shows the Fast Fourier Transforms of the third mass subject to a unit impulse applied at the fifth mass for a duration of $10 \mu \mathrm{s}$. The result indicates that, except some differences in magnitude, prediction for modal frequencies is the same regardless of the integration technique used. Furthermore, it is important to note that accumulation of numerical error using Houbolt integration method tends to be stabilizing, while two other integration schemes do not. Therefore, the Houbolt integration scheme was adapted into the proposed DT-LM-TMM approach.

By applying the recursive substitution process from the left and right boundaries to the central third mass subsystem, we obtain a reduced order model of the form (27). For this reduced order model, we assume that the position and velocity of the third mass is available for feedback and design an optimal linear quadratic 


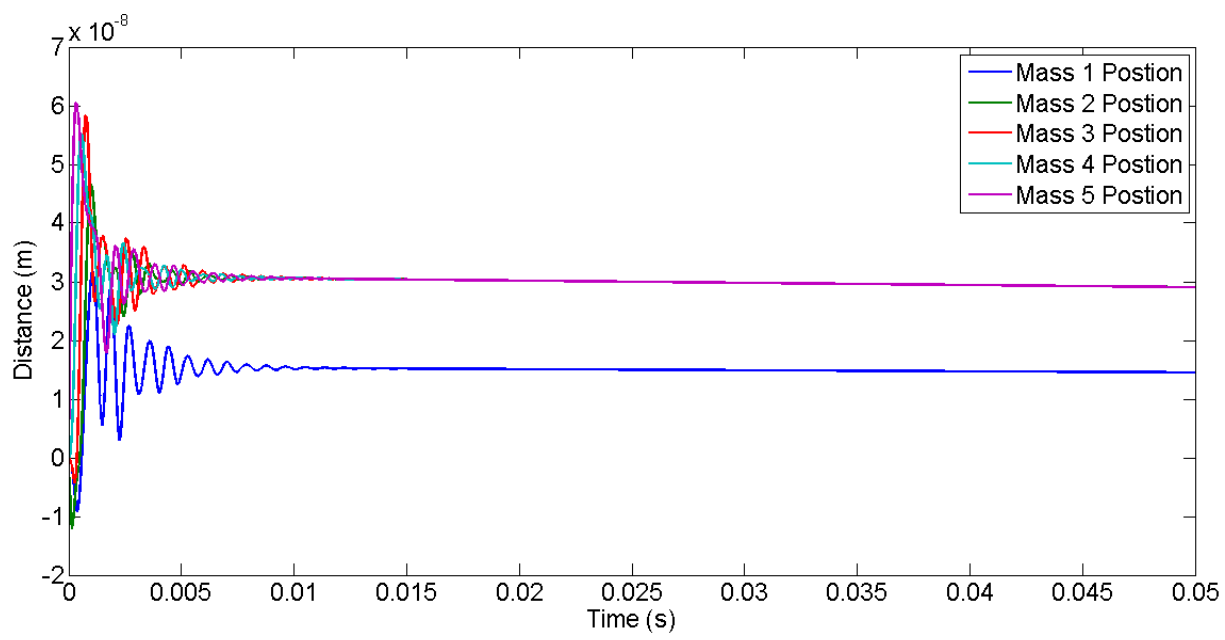

Figure 7. The continuous LQR controller synchronizes the four right masses first and then regulates all masses to zero.

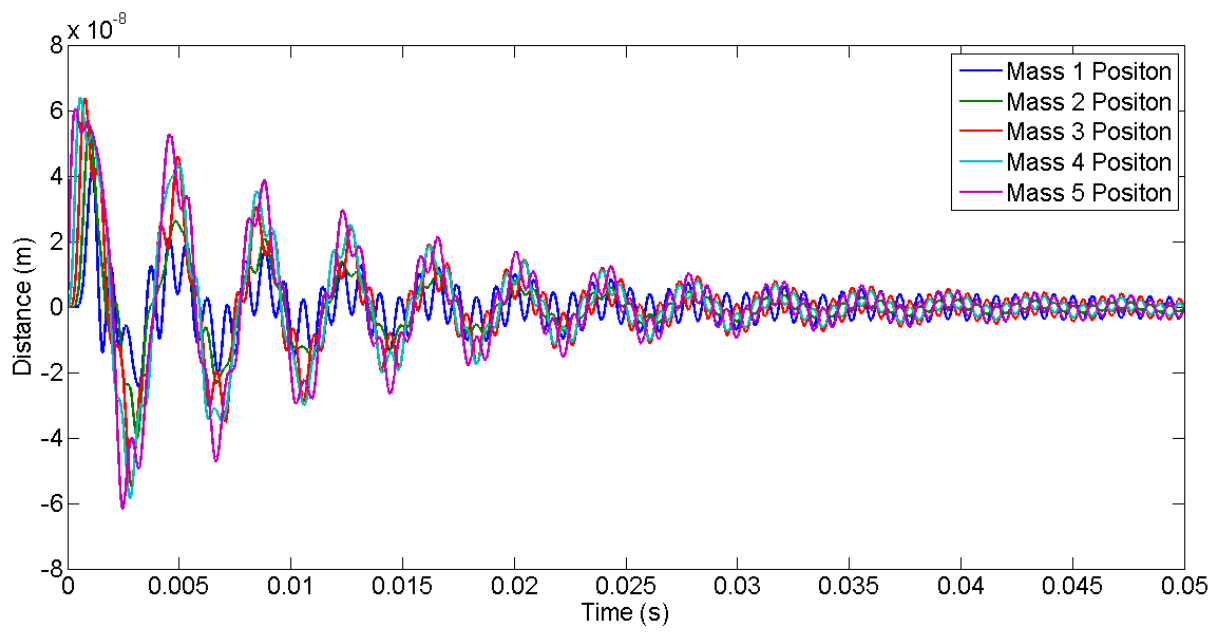

Figure 8. The DT-LM-TMM LQR controller regulates the controlled mass and to propagate to neighboring masses for suppression.

regulator (LQR) feedback control. The weighting matrices $Q$ and $R$ are chosen as follows,

$$
Q=\left[\begin{array}{ccc}
1 e^{9} & 0 & 0 \\
0 & 1 & 0 \\
0 & 0 & 1
\end{array}\right], R=0.01
$$

It can be shown that the optimal controller developed for the reduced order system is able to suppress all the states. To highlight this observation, and for the purpose of comparison, we have also developed a continuous-time full state optimal LQR controller with the same choice of weighting matrices (30). The simulations for both full state and reduced order LQR controlled system are performed in MATLAB environment. Figure 6 shows the comparison of the total system energy for two approaches, and it indicates that they both are able to suppress overall vibrational motions with comparable performance. The average system energy over the simulation time for the full state continuous LQR is $8.923 e^{-11}$ and for DT-LM-TMM is $1.113 e^{-11}$.

Though both LQR controllers are able to stabilize overall dynamics, the response of each mass behaves very differently for each feedback-controlled system. The full state continuous-time LQR controller tends to first kill off all the low frequency content and the primary modes by forcing the four right masses to

$$
9 \text { of } 12
$$


Table 1. Parameters for simulated lumped mass system.

\begin{tabular}{ll} 
Parameter & Value \\
\hline Number of Masses & 5 \\
Mass & $0.0323 \mathrm{~kg}$ \\
Spring Stiffness & $1000000 \frac{\mathrm{N}}{\mathrm{m}}$ \\
Damping Constant & $1 e^{-7} \frac{\mathrm{N}}{\mathrm{ms}}$ \\
Time Step & $10 \mu \mathrm{s}$
\end{tabular}

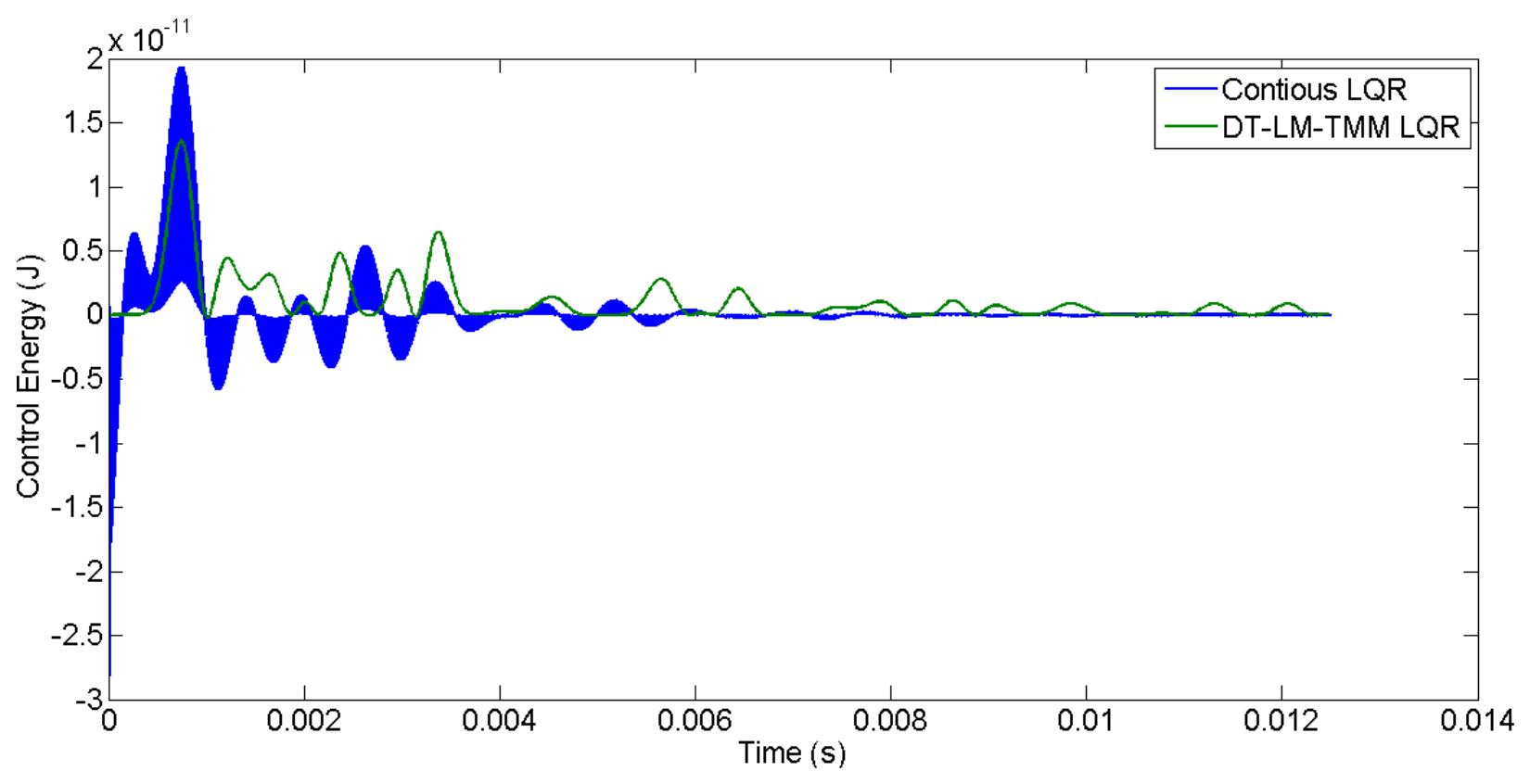

Figure 9. The control energy of the system for the full state continuous LQR and the DT-LM-TMM LQR.

synchronize, then slowly regulates them to zero. This can be seen in Figure 7 On the other hand, the DT-LM-TMM LQR controller simply attempts to attenuate the position and velocity of the controlled mass (third mass) and this effect is then propagated through boundary elements to achieve overall suppression, as shown in Figure 8.

The amount of energy that is needed to regulate the vibrational motions is an important consideration when comparing the performance of controllers. Figure 9 shows the control energy of the two controllers. The control energy is calculated by multiplying the force input and the distance the mass traveled in the time step that the force was applied. If the mass is moving in the same direction as the direction of applied force, then the input energy in Figure 9 is positive, it is negative if the mass is traveling in the opposite direction of force. We can see that with the full state knowledge the continuous LQR has many occasions where it is attempting to simply slow down the system (negative energy), while the DT-LM-TMM always utilizes positive energy. This suggests that DT-LM-TMM always utilize the control force $f_{n}$ along the motion of central mass or when it is greater than the exogenous input $\alpha$.

Figure 9 also shows one of the advantages of the DT-LM-TMM approach, in that we can see the full state continuous LQR controller tends to oscillate at very high frequencies, resulting in the nearly shaded in blue sections. In these simulations the highest frequency content of the full state continuous LQR is $1.37 \mathrm{Mhz}$, whereas the DT-LM-TMM is about 1.6Khz. Though both are too high for any practical actuation purpose, but by selecting the time step of $10 \mu s$ we have set a hard cap on the bandwidth at 10Khz. We believe the frequency content, especially in the case of DT-LM-TMM, can be reduced substantially by simply loosing up the integration time step without compromising too much on the numerical accuracy.

The analyses performed thus far were derived from the choice of weighting matrices given in (30). In 


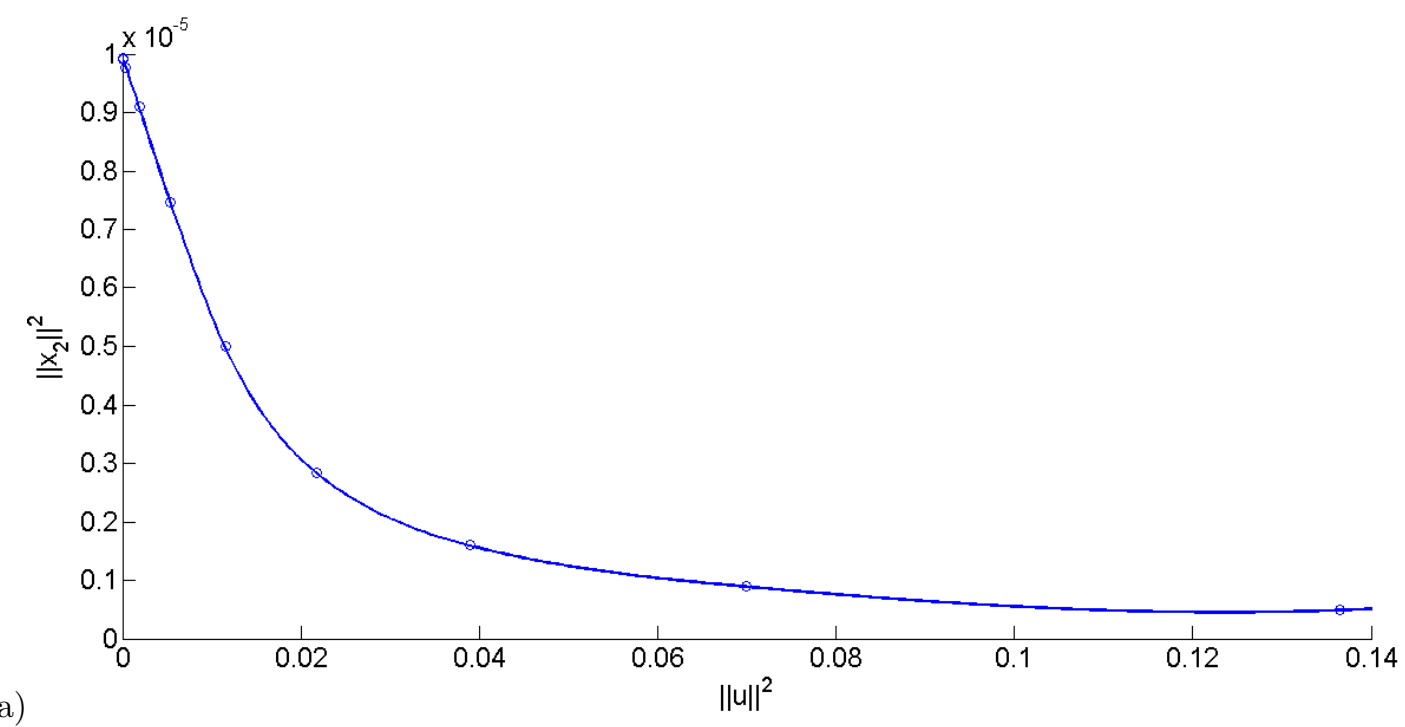

a)

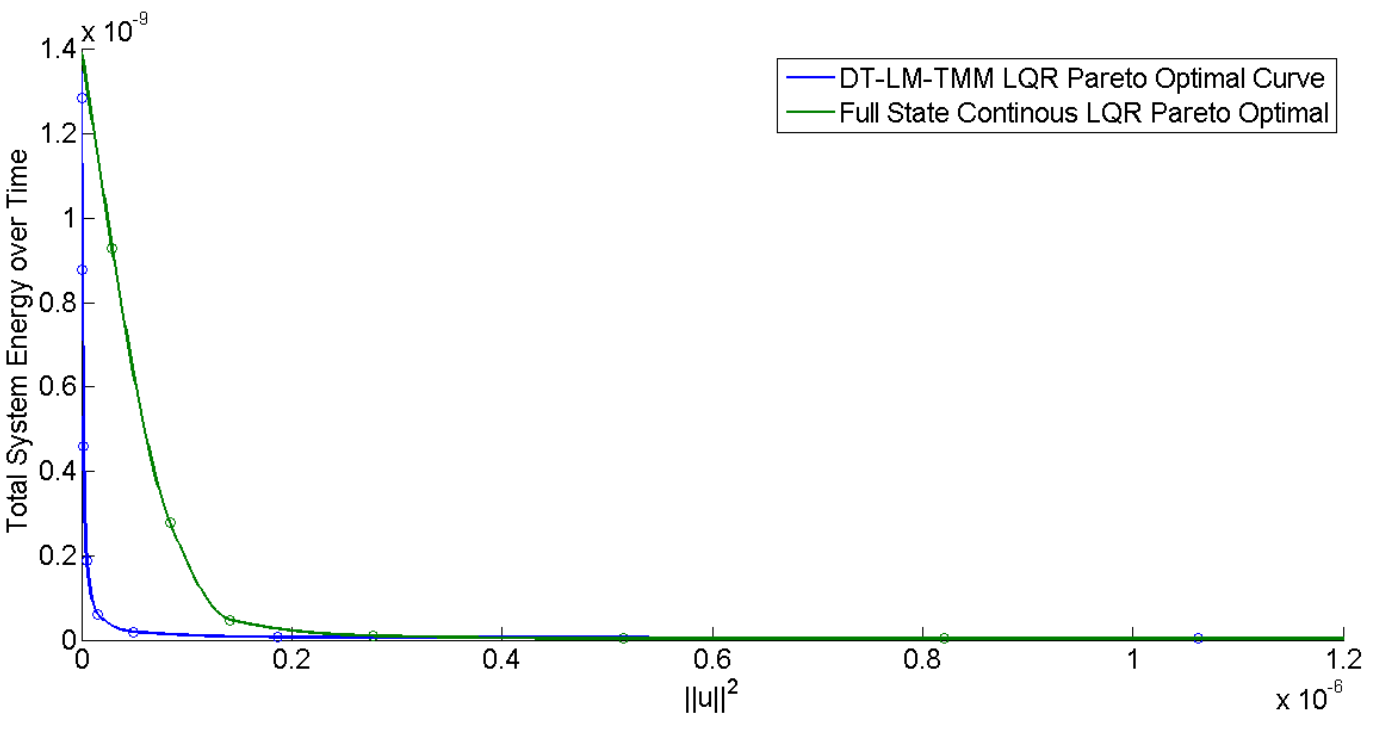

Figure 10. Closed-loop performance vs. control energy: (a) Pareto optimal curve for the reduced order model through DT-LM-TMM; (b) Pareto optimal curves for the whole system under the same set of simulations as in (a) and for the full state continuous LQR simulations.

Figure 10, we show the Pareto optimal curves of the LQR control design with the reduced order model through DT-LM-TMM over a range of $Q$ and $R$ selections. The plots offer an interesting and important control design trade-off, from which one may determine the "optimal" design point that renders the best closed-loop performance given the available control effort. We also compare the Pareto optimal curves of the DT-LM-TMM LQR controller with that of the full state continuous LQR controller and observe that within a reasonable range of $Q$ and $R$ both controllers render about the same level of performance.

\section{Conclusions}

In this paper, we have confirmed the viability of the proposed DT-LM-TMM approach as a simulation technique, which provided a means of calculating the propagation of boundary conditions for lattice-based structures. Furthermore, the proposed approach renders itself naturally to be formulated as a decentralized control problem for a reduced order model of much lower dimension. A stabilizing controller utilizing LQR approach was then applied to this control-centric reduced-order model, and we observed that the performance was similar to, if not better than, the performance of a continuous-time full state feedback LQR controller 
applied to the full order system. The advantages of proposed methodology were apparent, in that we only need to deal with a localized model of small number of states and need to feedback only local states, hence less sensor knowledge. In addition, we gain many advantages by utilizing the discrete-time approach, such as easy migration to flight control software and explicit control with maximum bandwidth.

\section{Acknowledgments}

N. Cramer and M. Teodorescu would like to thank UARC, ASL, and NASA Ames Research Center for sponsoring their research, and S. Swei and K. Cheung would like to thank NASA ARMD Seedling Team Project for supporting their research.

\section{References}

\footnotetext{
${ }^{1}$ Nguyen, N. and Urnes, J., "Aeroelastic Modeling of Elastically Shaped Aircraft Concept via Wing Shaping Control for Drag Reduction," AIAA Atmospheric Flight Mechanics Conference, 2012, pp. 13-16.

${ }^{2}$ Swei, S. S.-M. and Nguyen, N., "Aeroelastic Wing Shaping Control Subject to Actuation Constraints," 2014.

${ }^{3}$ Nguyen, N., "Elastically Shaped Future Air Vehicle Concept," NASA Innovation Fund Award, 2010.

${ }^{4}$ Urnes, J., Nguyen, N., Ippolito, C., Totah, J., Trinh, K., and Ting, E., "A Mission Adaptive Variable Camber Flap Control System to Optimize High Lift and Cruise Lift to Drag Ratios of Future N+3 Transport Aircraft," 51st AIAA Aerospace Sciences Meeting, Grapevine, TX, Janurary, 2013.

${ }^{5}$ Xiong, J., Ma, L., Wu, L., Wang, B., and Vaziri, A., "Fabrication and crushing behavior of low density carbon fiber composite pyramidal truss structures," Composite Structures, Vol. 92, No. 11, 2010, pp. 2695-2702.

${ }^{6}$ Vasiliev, V. and Razin, A., "Anisogrid composite lattice structures for spacecraft and aircraft applications," Composite Structures, Vol. 76, No. 1, 2006, pp. 182-189.

${ }^{7}$ T.M. Tan, A. Yousuff, L. B. and Konstantinidis, M., "A modified finite element-transfer matrix for control design of space structures," Computers and Structures, Vol. 36, No. 1, 1990, pp. $47-55$.

${ }^{8}$ Kumar, A. S. and Sankar, T., "A new transfer matrix method for response analysis of large dynamic systems," Computers Es structures, Vol. 23, No. 4, 1986, pp. 545-552.

${ }^{9}$ Evans, A. G., Hutchinson, J., and Ashby, M., "Multifunctionality of cellular metal systems," Progress in Materials Science, Vol. 43, No. 3, 1998, pp. 171-221.

${ }^{10}$ Siljak, D. D., Large-scale dynamic systems: stability and structure, Vol. 310, North-Holland New York, 1978.
} 